\title{
The Influence of the Gas-Distributing Grid Diameter on the Transition Velocity and Hydrodynamics of the Bottom Layer in Circulating Fluidized Bed Installations
}

\author{
V. G. Tuponogov and A. P. Baskakov \\ Ural Federal University, ul. Mira 19, Yekaterinburg, 620002 Russia
}

\begin{abstract}
The dependences of dimensionless fluidization velocities separating bubble, transition, and fast fluidization regimes on the properties of dispersed material for particles belonging to groups B and D (according to D. Geldart's classification) are presented. Correspondence between the considered dependences and experimental data obtained by different researchers and their correlation with critical fluidization velocities and particle terminal velocities are shown. The hydrodynamic mechanisms governing the saturation of fluidized bed with bubbles on reaching the transition fluidization velocity in installations having different sizes are considered. Factors due to which a bottom bubble layer disappears in narrow installations and is retained on large-diameter grids in an intense channel forming mode are explained. Experimental data are presented from which it is seen that the bubble layer hydrodynamics depends on the gas-distributing grid diameter and that this diameter has an insignificant influence on the fluidization velocity during the transition from a bubble to fast fluidization regime.
\end{abstract}

Keywords: fluidization regimes, transition velocities, bottom layer, bubbles, gas-distributing grid

DOI: $10.1134 / \mathrm{S} 0040601513110116$

In the mid 1970s, a few companies in Finland, Federal Republic of Germany, and the United States used the entrainment of particles from a fluidized bed as a basis of a new technology. This idea was in contrast to the approach that existed at that time, according to which the above-mentioned entrainment was regarded as a negative phenomenon. Instead of limiting the fluidization velocity in a stationary bubble fluidized bed within the range $1-4 \mathrm{~m} / \mathrm{s}$, this velocity was increased in new installations to $6 \mathrm{~m} / \mathrm{s}$. In addition, recirculation of entrained material was organized. Turbulent mixing of air, fuel, and inert particles that takes place during the firing of fuel in such installations with a circulating fluidized bed (CFB) has a stabilizing effect on the combustion process and on the bed temperature. CFB furnaces and boiler units have been widely used in developed countries in working out clean technologies for firing solid fuels; the unit capacity of CFB boilers reaches $1000 \mathrm{MW}$. The strategy for development of Russia's power industry supposes, in particular, the use of environmentally safe CFB technologies for different applications, including combined-cycle plants with integrated-cycle gasification and thermochemical treatment of coal [1].

Specialists who carry out CFB investigations pay the greatest attention to studying the hydrodynamic properties of the bed, which determine the regularities pertinent to the heat-and-mass transfer processes in the installation and reliability of equipment operation [2]. This article addresses the regularities pertinent to hydrodynamics under the conditions of high-velocity fluidization regimes connected with the bottom layer becoming saturated with bubbles, and which in some cases cause the layer to disappear.

According to the currently existing ideas about the fluidized bed dynamics, four fluidization regimes are distinguished, which depend on fluidization velocity $u$ : bubble, transitional, turbulent, and forced regimes. As is shown in Fig. 1 [3], the velocity corresponding to the onset of a transitional regime $u_{\mathrm{c}}$ is determined from the maximum of pressure pulsation amplitude (the rms deviation $\sigma_{p}$ ), and the velocity $u_{\mathrm{k}}$ at which the onset of a steady turbulent regime occurs corresponds to the end of the pressure pulsation amplitude dropping segment. The transport velocity $u_{\mathrm{tr}}$ separating the turbulent regime from the fast regime may exceed the critical fluidization velocity $u_{\mathrm{mf}}$ by a factor of $1000-$ 1500 for fine particles (group A according to Geldart's classification) and by several tens of times for larger particles (group B). For the turbulent and fast fluidization regimes used in CFB installations, recirculation of material is a must. When a CFB installation operates in the fast regime, the flow density in the installation's riser leg depends, unlike that in the turbulent regime, on the flowrate of circulating material serving to maintain the flow mass.

The performed in [3] processing of experimental data obtained in installations fitted with a gas-distributing grid with diameter $D=0.05-0.8 \mathrm{~m}$ with the use 


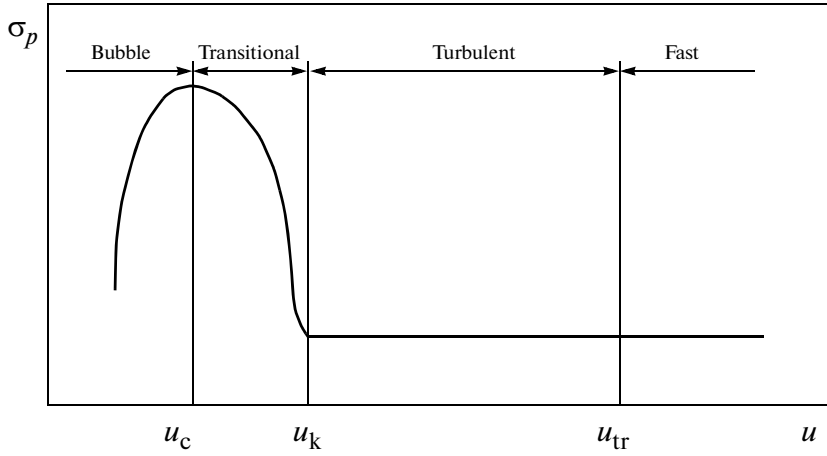

Fig. 1. Dependence of pressure fluctuation amplitude in fluidized bed installations on fluidization velocity in different fluidization regimes [3].

of 99 kinds of particles (the diameters of particles were varied in the range $d_{\mathrm{p}}=22-1200 \mu \mathrm{m}$ and their density was $\rho_{\mathrm{p}}=327-4510 \mathrm{~kg} / \mathrm{m}^{3}$ ) showed the following. A steady turbulent regime is obtained only with group A particles, where as for particles of groups B and D, which just encompass the particles of fuel and inert material in CFB furnaces, the velocities $u_{\mathrm{k}}$ and $u_{\mathrm{tr}}$ are equal to each other; i.e., the transitional regime transfers into the fast regime without any intermediate state.

Our experimental data on the transition velocity $u_{\mathrm{c}}$ and the data of other researchers [3] are described most accurately by the following relation [4] (Fig. 2):

$$
\operatorname{Re}_{\mathrm{c}}=0.633 \mathrm{Ar}^{0.467},
$$

where the Reynolds number $\operatorname{Re}_{\mathrm{c}}=\frac{u_{\mathrm{c}} d_{\mathrm{p}}}{v_{\mathrm{g}}} ; v_{\mathrm{g}}$ is the gas kinematic viscosity coefficient, $\operatorname{Ar}=\frac{g d_{\mathrm{p}}^{3}\left(\rho_{\mathrm{p}}-\rho_{\mathrm{g}}\right)}{v_{\mathrm{g}}^{2} \rho_{\mathrm{g}}}$ is the Archimedean number, and $g$ is the acceleration of gravity.

The experimental values of velocity corresponding to the shift to the turbulent regime $u_{\mathrm{k} \text {.tr }}$ obtained for group B particles (there were 39 fractions of the following materials: sand with $d_{\mathrm{p}}=60-1200 \mu \mathrm{m}$, coal with $d_{\mathrm{p}}=730-1030 \mu \mathrm{m}$, glass with $d_{\mathrm{p}}=65-650 \mu \mathrm{m}$, iron ore with $d_{\mathrm{p}}=56-105 \mu \mathrm{m}$, and silica gel with $d_{\mathrm{p}}=$ $476-1057 \mu \mathrm{m}$ ) in installations fitted with a grid having a diameter from 78 to $380 \mathrm{~mm}$ are described by the following expression:

$$
\operatorname{Re}_{\mathrm{k}}=\operatorname{Re}_{\mathrm{tr}}=1.45 \mathrm{Ar}^{0.484},
$$

where the Reynolds numbers $\operatorname{Re}_{\mathrm{k}}$ and $\mathrm{Re}_{\mathrm{tr}}$ correspond to the velocities $u_{\mathrm{k}}$ and $u_{\mathrm{tr}}$.

Dependences of the Reynolds numbers on the Ar number (a so-called chart of fluidization regimes [3] were constructed (Fig. 3) from formulas (1) and (2) and

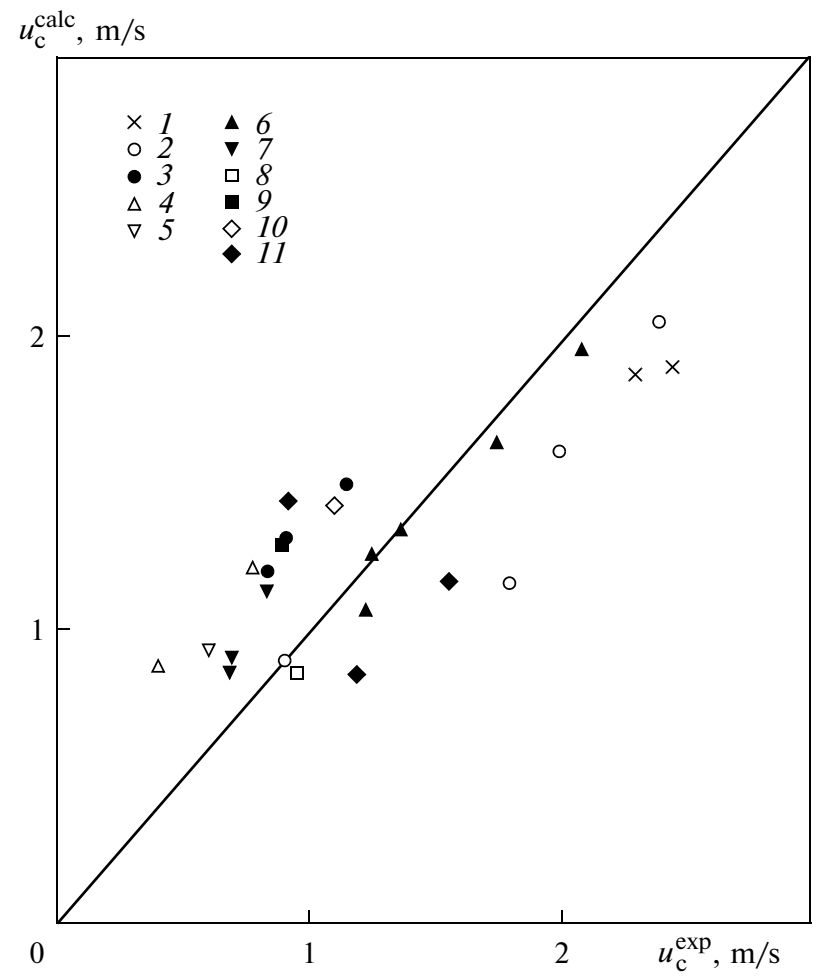

Fig. 2. Comparison between the values of transition fluidization velocity $u_{\mathrm{c}}$ calculated from (1) and experimental data. (1) Data obtained by the authors for corundum particles, $d_{\mathrm{p}}=0.418 \mathrm{~mm}$ and $D=0.2$ and $0.25 \mathrm{~mm}$. Data of [3]: (2) vermiculite, aluminum, $d_{\mathrm{p}}=0.042-0.223 \mathrm{~mm}$ and $D=0.076$ and $0.152 \mathrm{~m}$; (3) coal, $d_{\mathrm{p}}=0.73 \mathrm{~mm}$; (4) aluminosilicate FCC (fluid catalytic cracking), $d_{\mathrm{p}}=0.067 \mathrm{~mm}$ and $D=0.224 \mathrm{~m}$; (5) polyetylene, $d_{\mathrm{p}}=0.325 \mathrm{~mm}$ and $d=$ $0.102 \mathrm{~m} ;(6)$ alumosilicate FCC, sand $d_{\mathrm{p}}=0.06-1.2 \mathrm{~mm}$ and $D=0.092 \mathrm{~m} ;(7)$ alumosilicate FCC, sand $d_{\mathrm{p}}=0.078$ and $0.13 \mathrm{~mm}$ and $D=0.682$ and $6.2 \mathrm{~m} ;(8)$ alumosilicate FCC, catalyst, $d_{\mathrm{p}}=0.056-0.134 \mathrm{~mm}$ and $D=0.05 \mathrm{~m}$; (9) glass balls, $d_{\mathrm{p}}=0.362 \mathrm{~mm}$ and $D=0.1 \mathrm{~m}$; (10) sand, $d_{\mathrm{p}}=0.43 \mathrm{~mm}$ and $D=0.38 \mathrm{~m} ;(11)$ sand, $d_{\mathrm{p}}=0.268 \mathrm{~mm}$; aluminum, $d_{\mathrm{p}}=0.103 \mathrm{~mm}$, glass, $d_{\mathrm{p}}=0.157 \mathrm{~mm}$.

from the expression for the Reynolds number determining the minimal fluidization velocity $\mathrm{Re}_{\mathrm{mf}}[5]$ :

$$
\operatorname{Re}_{m f}=(1135.7+0.0408 \mathrm{Ar})^{0.5}-33.7 \text {, }
$$

The dependence for dimensionless terminal velocity [6] is also plotted in this figure:

$$
\operatorname{Re}_{\mathrm{t}}=\frac{\mathrm{Ar}}{18+0.61 \sqrt{\mathrm{Ar}}} .
$$

The graphs shown in Fig. 3 indirectly (in terms of the relevant Reynolds numbers) illustrate the transition velocities as functions of the physical properties of particles, as well as a correlation between the transition velocities and the minimal fluidization and terminal velocities for particles of groups $B$ and $D$. For large particles $\left(\mathrm{Ar} \approx 10^{5}\right)$ the velocity $u_{\mathrm{k} . \mathrm{tr}}$ is equal to the terminal velocity $u_{\mathrm{t}}[6]$. 


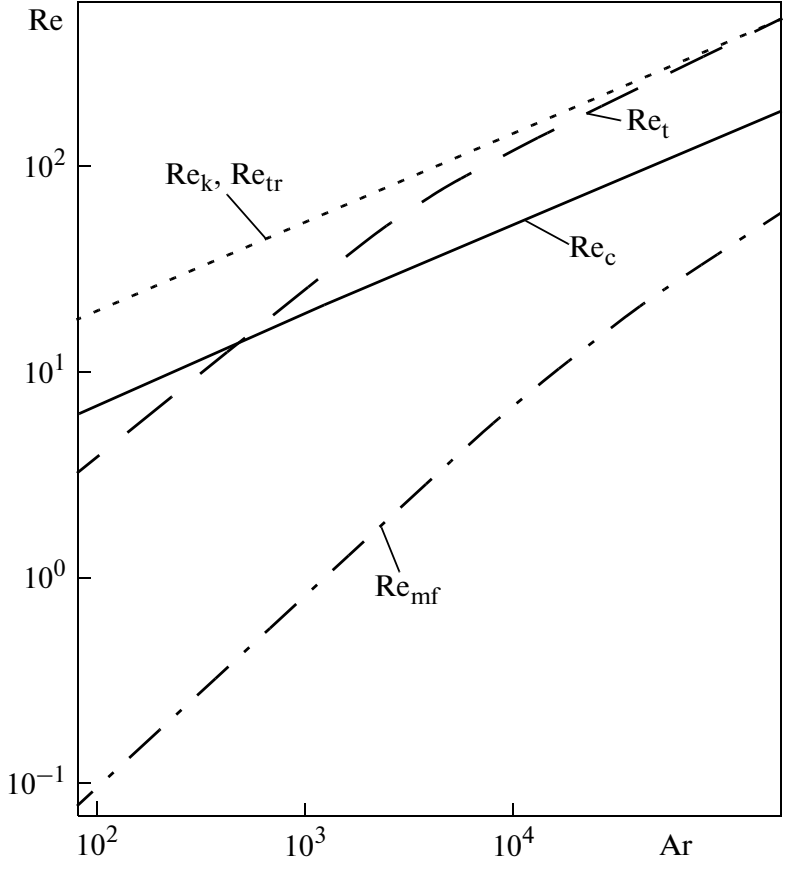

Fig. 3. Chart of fluidization regimes [formulas (1)-(4)].

One matter that is of importance in organizing the combustion and heat transfer processes in the furnaces of CFB boilers is to predict the change in the bed structure when a transition occurs from the bubble to fast fluidization regime, during which the shape of bubbles becomes unstable and the bubbles collapse. The majority of researchers relate the collapse of bubbles to achieving the state in which a bubble reaches its maximal (critical) diameter $D_{\text {bub }}^{\max }$ at its maximal velocity. The gas circulation velocity in a bubble is approximately equal to the bubble motion velocity with respect to particles $u_{\mathrm{bub}}$. With a growth of the fluidization velocity $u, u_{\text {bub }}$ can reach the particle terminal velocity $u_{\mathrm{t}}$ and even exceed it. In this case, particles from the stern zone are entrained by circulating gas to inside the bubble, and the bubble collapses. However, the growth of bubble diameter $D_{\text {bub }}$ (and its velocity) can be limited by the installation sizes. According to the data of studies reported in [7], the influence of the installation walls becomes noticeable at $D_{\text {bub }} / D=0.3$. Therefore, with decreasing the diameter $D$ of the installation's gas-distributing grid, bubbles "overfill" the bed at a smaller value of fluidization velocity $u$; i.e., the transition velocity $u_{\mathrm{c}}$ becomes smaller. At the same time, the smaller the installation diameter, the lower the bubble absolute velocity $u_{\mathrm{a}}$ determined from the expression $u_{\mathrm{a}}=u-u_{\mathrm{mf}}+0.35 \sqrt{g D}$, and in a larger-size installation the bubble critical velocity can be obtained at a lower value of fluidization velocity $u$. Hence, a change of the grid diameter in small-size installations entails two opposite effects: on one hand, as the grid diameter decreases, so does the velocity $u_{\mathrm{c}}$ because the bed is saturated with bubbles more rapidly, and on the other hand, this velocity increases due to a decrease of the bubble velocity $u_{\mathrm{a}}$. As a result, the effect the grid diameter has on the velocity $u_{\mathrm{c}}$ becomes ambiguous in nature. At the same time, the settling of a turbulent fluidization regime in small installations having grids with a diameter commensurable with the maximal diameters of bubbles can be determined from the fact that the bottom bubble layer smears and then disappears at fluidization velocities exceeding $u_{\mathrm{c}}$.

With the bed entire volume completely filled with bubbles (a dense cubic packing of identical spheres), the bed porosity is around 0.71 . Investigations show that in installations with a large-area gas-distributing grid (in which $D_{\text {bub }} / D<0.3$ ), bubbles are distributed over the bed nonuniformly, and at high fluidization velocities they merge into separate channels via which gas flows are breaking through. These channels are formed as a result of coalescence (merging) and acceleration of bubbles in a vertical chain. When two ascending bubbles merge with each other, the velocity of the lower overtaking bubble increases with respect to particles by almost a factor of 2 as it enters in the wake zone of the upper (overtaken) bubble (the diameter of this zone is approximately equal to $1.2 D_{\text {bub }}$, and the absolute velocity increases in this case to $u_{\mathrm{a}}=u=$ $u_{\mathrm{mf}}+2 u_{\text {bub }}$ ) because the velocity of material particles in the wake zone is close to $u_{\text {bub }}$. At fluidization velocities $u>u_{\mathrm{c}}$ large bubbles may also disintegrate into smaller ones, a phenomenon the authors observed in experiments carried out on transparent models [8]. In [9], pressure fluctuations in the bed occurring during the transition from a bubble to turbulent mode were modeled in installations fitted with a large-diameter grid $\left(D>>D_{\text {bub }}\right)$. In the first version of modeling the initial conditions, calculations were carried out on the assumption that, starting with a certain height above the gas-distributing grid, bubbles that have reached the maximal diameter $D_{\text {bub }}^{\max }$ do not collapse (and do not grow), but the distance between them decreases due to an increase of $u$. In the second version of modeling the initial conditions, it was assumed that on having reached $D_{\text {bub }}^{\max }$, bubbles disintegrated into smaller ones. In both versions, maximums of pressure pulsation amplitude were obtained at the fluidization velocity corresponding to $D_{\text {bub }}^{\max }$, and the value of transition velocity was in full agreement with the experimental values of $u_{\mathrm{c}}$ (the experimental installation's maximal diameter $\mathrm{D}$ was equal to $1.56 \mathrm{~m}$ ). A conclusion can be drawn from the simulation results [9] that after reaching the transition velocity $u_{\mathrm{c}}$ (and the maximal diameter of bubbles), pressure pulsations in the bed decrease irrespectively of whether or not bubbles collapse after that; the most important is that they do not grow any more. 


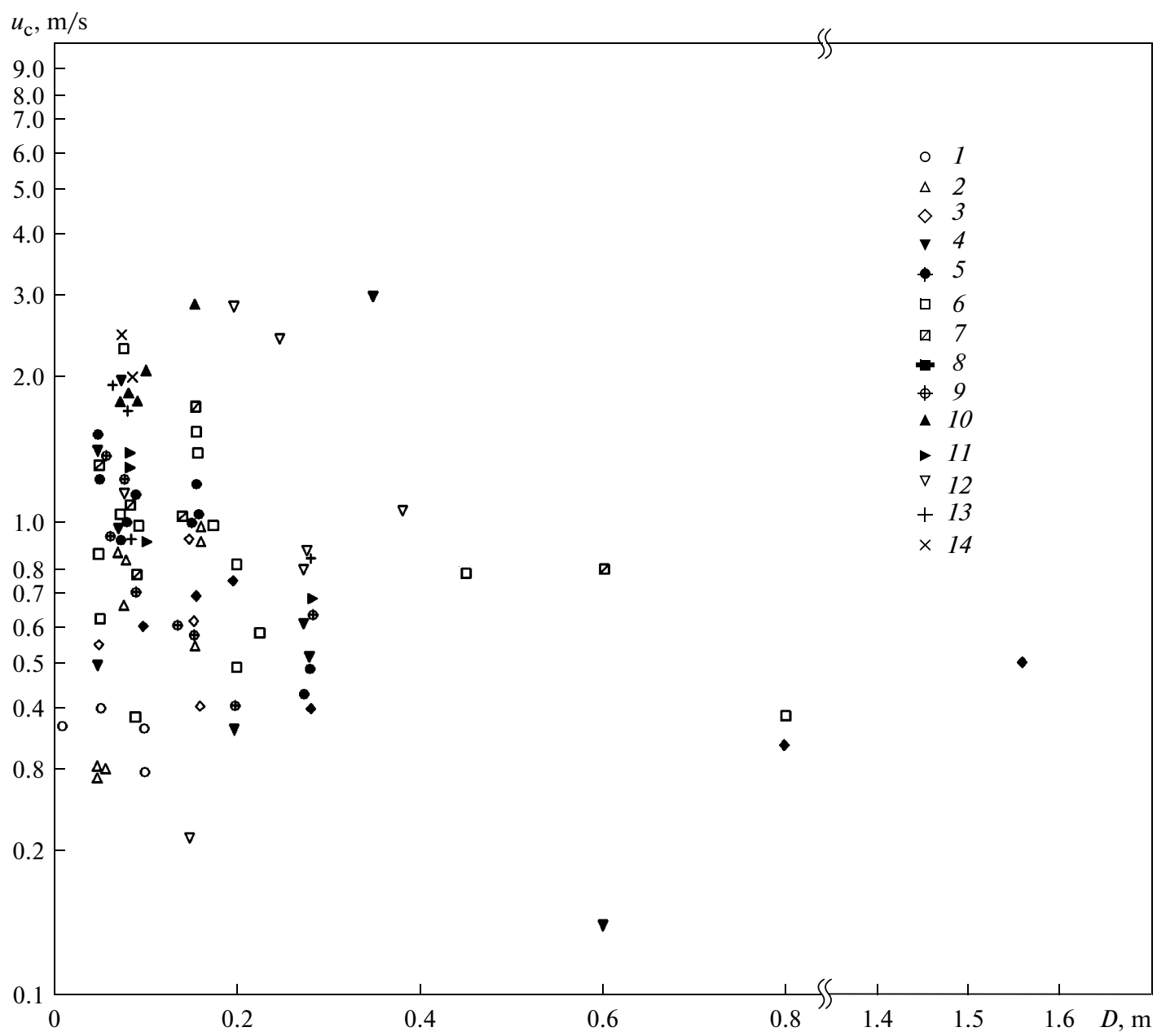

Fig. 4. Dependence of transition velocity $u_{\mathrm{c}}$ on the gas-distributing grid diameter. Ranges of the Ar number: (1) $0.72-0.87,(2)$ $2.15-3.90$, (3) 4.52-6.30, (4) 7.3-8.6, (5) 9.71-11.50, (6) 16.5-24.7, (7) 31.5-47.8, (8) 111-130, (9) 208-557, (10) $1.6 \times 10^{3}-$ $2.3 \times 10^{3},(11) 3.12510^{3}-5.53 \times 10^{3}$, (12) $7.52 \times 10^{3}-9.99 \times 10^{3}$, (13) $1.96 \times 10^{4}-3.58 \times 10^{4}$, and (14) $9.54 \times 10^{4}-12.4 \times 10^{4}$.

Figure 4 shows the study results reported in $[3,9$, 10] and the data obtained by the authors (see Fig. 2), which are grouped for narrow intervals of the Ar number. The majority of experimental points $(1,2,8,9,10$, $11,13,14)$ were obtained in installations with $D \leq 0.3 \mathrm{~m}$. Of these, the velocity $u_{\mathrm{c}}$ of particle group (2) increases with increasing the diameter; the same of particle groups $(1,8,9,11,13,14)$ decreases, and the velocity of particle group (10) varies in different directions. The other points were obtained for $D>0.3 \mathrm{~m}$. Of them, $u_{\mathrm{c}}$ of one group (4) varies in different directions with increasing the diameter; in group (5) $u_{\mathrm{c}}$ remains almost unchanged, and in three groups $(6,7$, and 12$)$ it decreases. The data presented above show that an unequivocal dependence of $u_{\mathrm{c}}$ on $D$ does not exist, although a tendency toward decreasing the transition velocity $u_{\mathrm{c}}$ with increasing the apparatus diameter can be noticed.

Thus, it follows from what was said above that the transitional and fast fluidization regimes are realized in different ways depending on the installation diameter. In narrow apparatuses $\left(D<D_{\text {bub }}\right)$ the bed first becomes uniformly turbulent on reaching $u_{\mathrm{c}}$; then, on reaching the transport velocity $u_{\mathrm{tr}}$ it takes an annular structure with particles falling down, predominantly at the installation walls; as this takes place, the bottom layer disappears. In apparatuses with a large-diameter grid, the bottom layer is retained in the entire range of fluidization velocities, but at velocities $u>u_{\mathrm{c}}$ the ascending bubbles are transformed into through channels for the passage of gas. Results directly confirming this conclusion were obtained in the studies reported in $[11,12]$, in which for sand particles $\left(d_{\mathrm{p}}=0.31 \mathrm{~mm}\right.$, $u_{\mathrm{mf}}=0.08 \mathrm{~m} / \mathrm{s}$, and $u_{\mathrm{tr}}=2 \mathrm{~m} / \mathrm{s}$ ) in an installation with a gris measuring $0.7 \times 0.12 \mathrm{~m}$ in size the moment at which one regime began to replace another one was observed at $u_{\mathrm{c}}=1.2 \mathrm{~m} / \mathrm{s}$ (which corresponded to the maximum of $\sigma_{\mathrm{p}}$ ), and at velocity $u_{\mathrm{tr}} \cong 4 \mathrm{~m} / \mathrm{s}$ and higher the bottom layer disappeared (it was carried upward and in a recirculation mode), whereas with the same 
material used in a CFB boiler fitted with a grid measuring $1.72 \times 1.44 \mathrm{~m}$ in size, a bottom layer existed in all fluidization regimes, including the fast regime at $u=4.5 \mathrm{~m} / \mathrm{s}$.

\section{CONCLUSIONS}

(1) The velocity $u_{\mathrm{c}}$ at which transition begins from a bubble to turbulent fluidization regime, defined by expression (1), corresponds to bubbles of the maximal sizes and to the maximal pressure pulsation amplitudes in the installation. For group B particles, the velocity at which a steady fast fluidization regime commences is almost identical with the terminal velocity in the range of Ar number from $10^{4}$ to $10^{5}$.

(2) The results obtained from experimental investigations carried out in installations fitted with gas-distributing grids of diameter $D=0.05-0.80 \mathrm{~m}$ show that the transition velocity $u_{\mathrm{c}}$ slightly decreases with increasing the grid diameter.

(3) At fluidization velocities exceeding the velocity of a steady turbulent mode $u_{\mathrm{k}}$, the bubble bottom layer in installations with small-diameter grids $\left(D<D_{\text {bub }}\right)$ smears and disappears. In installations with largediameter grids the bottom layer is retained due to intense formation of through channels for passage of excess gas.

\section{REFERENCES}

1. V. M. Batenin, Yu. A. Zeigarnik, and V. M. Maslennikov, "About the strategy for development of the russian power engineering (after ten years)," Therm. Eng., No. 4, 261 (2102).

2. G. A. Ryabov and O. M. Folomeev, "Substantiating the techniques for calculating the furnace circuit of circu- lating fluidized bed boilers,” Therm. Eng., No. 6, 456 (2011).

3. J. Arnaldos and J. Casal, "Prediction of transition velocities and hydrodynamical regimes in fluidized beds," Powder Technology 86, 285 (1996).

4. M. Nakajima, M. Harada, M. Asai, et al., "Bubble fraction and voidage in an emulsion phase in the transition to a turbulent fluidized bed," in Proceedings of the 3rd Conference on CFB Technology (Pergamon Press, Oxford, 1991), pp. 79-84.

5. Gas Fluidization Technology, Ed. by D. Geldart (J. Wiley \& Sons Ltd., Chichester, 1986).

6. O. M. Todes and O. B. Tsitovich, Granulated Fluidized Bed Apparatuses (Khimiya, Leningrad, 1981) [in Russian].

7. J. M. Matsen, "Scale-up of fluidized bed processes: principle and practice," Powder Technology 88, 237 (1996).

8. A. S. Zavarov, A. P. Baskakov, and S. M. Grachev, Chemicothermal Treatment in a Fluidized Bed (Mashinostroenie, Moscow, 1985) [in Russian].

9. A. Chen and H.T. Bi, "Pressure fluctuations and transition from bubbling to turbulent fluidization," Powder Technology 133, 237 (2003).

10. G. S. Lee and S. D. Kim, "Bed expansion characteristics and transition velocity in turbulent fluidized bed," Powder Technology 62, 207 (1990).

11. F. Johnsson, R. C. Zijerveld, J. C. Schouten, et al., "Characterization of regimes by time-series analysis of pressure fluctuations," Int. J. Multiphase Flow 26, 663 (2000).

12. W. Zhang, F. Johnsson, and B. Leckner, "Time-dependent behavior of particle flow in CFD boilers," in Proceedings of the Tenth Eng. Found. Conf. on Fluidization, Beijing, China, May 20-25, 2001, pp. 253-260.

Translated by $V$. Filatov 